\title{
Salicylic Acid Improves Rice Seed Germination under Induced Drought Stress
}

\author{
Ch. Muhammad Rafiq ${ }^{1}$, Qasim Raza ${ }^{*}$, Awais Riaz ${ }^{1}$, Misbah Hanif ${ }^{2}$, Wajiha Saeed ${ }^{2}$, Shawaiz Iqbal ${ }^{1}$, Tahir \\ Hussain Awan ${ }^{1}$, Syed Sultan Ali ${ }^{1}$ and Muhammad Sabar ${ }^{1}$
}

${ }^{1}$ Rice Research Institute, Kala Shah Kaku, Sheikhupura, Punjab, Pakistan; ${ }^{2}$ Department of Plant Breeding and Genetics, University of Agriculture Faisalabad, Pakistan.

Ch. Muhammad Rafiq and Qasim Raza contributed equally to this study.

\begin{abstract}
Rice is the staple food of more than 50\% of the world's population, and water scarcity is threating its sustainable production. Dry seeded rice (DSR) technology has water and labour saving advantages over conventional transplanting culture, however, poor crop establishment due to reduced germination in variable field conditions greatly hampers its large-scale adaption. To address subordinate germination issues, we investigated the effects of five salicylic acid (SA) concentrations $(0,75,150,225$ and $300 \mathrm{ppm})$ on polyethylene glycol (PEG) induced drought stress conditions $(0,-0.2,-0.4,-0.6$ and $-0.8 \mathrm{MPa})$. Highly significant $(p<0.01)$ effects of drought, SA and their interactions were observed on seed germination. Interestingly, varying SA concentrations imparted more pronounced effects under higher osmotic stress levels. Day-wise germination data indicated that the SA treatments alleviated inhibitory effects of different osmotic stress levels after 2-4 days of stress applications. Under medium to high osmotic stress levels, mean germination time, germination index and seed vigour index (SVI) of SA primed seeds were better than non-primed seeds. Remarkably, SVI of all SA concentrations under - $0.2 \mathrm{MPa}$ osmotic stress was surprisingly improved as compared with control and other osmotic stress levels, indicating a 'drought-escape strategy' in rice seeds under low osmotic stress level. Overall, our results indicated that seed priming with $225 \mathrm{ppm}$ SA concentration is ideal under all environments and should be recommended in DSR system to improve early crop establishment and sustainable production.

Received | February 21, 2021; Accepted | March 28, 2021; Published | June 05, 2021

*Correspondence | Qasim Raza, Rice Research Institute, Kala Shah Kaku, Sheikhupura, Punjab, Pakistan; Email: qasimnazami@gmail.com

Citation | Rafiq, C.M., Q. Raza, A. Riaz, M. Hanif, W. Saeed, S. Iqbal, T.H. Awan, S.S. Ali and M. Sabar. 2021. Salicylic acid improves rice seed germination under induced drought stress. Journal of Innovative Sciences, 7(1): 152-160.

DOI | https://dx.doi.org/10.17582/journal.jis/2021/7.1.152.160

Keywords | Salicylic acid, DSR, Germination, PEG, Seed treatment, Sustainable production
\end{abstract}

\section{Introduction}

$\mathrm{R}$ ice is one of the most important food commodities and provides major portion of daily calories requirement, especially in underdeveloped and developing countries (Fukagawa and Ziska, 2019). The world population is increasing at an exponential rate, demanding approximately $70 \%$ increase in food production to meet the food security targets by 2050 (Fróna et al., 2014). Water scarcity or drought is one of the biggest threats to food security worldwide and could reduce crop production by 50\% (Budak et al., 2015). Therefore, management of drought stress is crucial for successfully achieving the desired production targets. About $77 \%$ of the total cultivated rice is conventionally transplanted globally 
(Rao et al., 2017). Nevertheless, the flood irrigation culture requires large amount of water, hence, this conventional establishment method is no longer suitable for sustainable rice production. Dry seeded rice (DSR) technology has emerged as an alternate to conventional transplanting culture (Liu et al., 2015a). The main advantages of DSR includes resource conservation (water saving) and economical, making it more suitable for sustainable rice production (Iqbal et al.,2019,2021). However, DSR confronts with poor crop establishment due to unequal water distribution in variable field conditions, which greatly hampers large-scale adoption of this technology. Thus, an intervention in current DSR technology is essentially needed to accelerate its large-scale adoption among farming community.

Seed germination starts after imbibition with water, initiation of embryo growth, changes in seed water content and completes with radicle emergence and elongation (Ali and Elozeiri, 2017). Among different abiotic factors which effect seed germination, temperature, drought, salinity and phytohormones have been reported to have massive influence on rice seed germination (Kurniasih et al., 2013; ElMokhtar et al., 2015; Jan et al., 2015; Liu et al., 2015b; Wei et al., 2015; Zheng et al., 2016). E1Mokhtar et al. (2015) studied the response of three rice cultivars to different salinity stress levels and reported highly significant effects of salt stress on rice seed germination. Similarly, Liu et al. (2015b) also reported highly significant effects of high temperature stress on rice seed germination. Water deficiency or drought significantly affects germination and seedling emergence in rice (Hussain et al., 2017; Islam et al., 2018). Water stress ceases metabolic activities in seeds, delays germination process and reduces the biomass production in germinating seeds, ultimately leading to poor crop establishment. To overcome these issues, farmers in Asia prefer to pre-treat seed before sowing to obtain uniform germination in DSR system (Zheng et al., 2016). Nevertheless, such interventions exhibited no beneficial effects under drought conditions (Matsushima and Sakagami, 2013).

Pre-treatment of crop seed with different agents could improve its germination. Among different pre-treatment approaches, seed priming is the most potent approach for improving synchronization of germination (Hussain et al., 2017). In rice, seed priming improves germination and seedling growth under abiotic stresses such as chilling, drought and salinity (Zheng et al., 2016; Sheteiwy et al., 2017; Shatpathy et al., 2018; Sarfraz et al., 2019; Sharma and Parikh, 2020). Salicylic acid (SA) is a naturally occurring phenolic compound which exists in plants at a very low concentration. This hormone like compound plays pivotal role in regulating the growth and development of plants. Earlier evidences also suggest that SA improves tolerance against biotic and abiotic stresses (Rivas-san and Plasencia, 2011; Khan et al., 2019; Mohamed et al., 2020). In rice, SA promotes seed germination under abiotic stresses such as salinity, cold and heavy metal stresses (Guo et al., 2007; Tavares et al., 2014; Wang et al., 2016). However, effect of seed priming with salicylic acid (SA) under drought stress is somewhat unclear in rice. In this study, we investigated effect of different SA concentrations on rice seed germination under induced osmotic stress conditions. Furthermore, we also examined effect of SA concentrations on seed vigour index.

\section{Materials and Methods}

\subsection{Experiment location and plant material}

This study was conducted under controlled laboratory condition at Rice Research Institute, Kala Shah Kaku (RRI, KSK), Pakistan to investigate the effects of drought and salicylic acid on rice seed germination. Seed of PK 1121 aromatic (a long grain and short stature aromatic rice variety) was collected from RRI, KSK and used for germination experiment.

\subsection{Pre-treatments and experiment setup}

Homogenous seeds with uniform colour, shape and size were selected and experiment was carried out with healthy seeds only. The petri dishes and seeds were disinfected with 10\% Sodium Hypochlorite $(\mathrm{NaOCl}$, Sigma Aldrich) solution for ten minutes, and then thoroughly rinsed with distilled water to remove all chlorine traces. The sterilized seeds were divided into five sets and separately soaked overnight in 0 (control), 75, 150, 225 and 300 ppm SA solutions (acetylsalicylic acid/Aspirin) at $35^{\circ} \mathrm{C}$, respectively. Afterwards, hydrated seeds were kept between two layers of blotting paper for surface drying. Then, surface dried seeds were transferred to sterilized petri dishes.

Five osmotic stress solutions $(0,-0.2,-0.4,-0.6$ and 
$-0.8 \mathrm{MPa}$ ) were prepared by dissolving $118,175,212$ and $254 \mathrm{~g}$ of polyethylene glycol (PEG) 6000 in 1000 $\mathrm{mL}$ of distilled water, respectively. Thirty pre-treated seeds in each treatment were allowed to germinate on saturated tissue papers in petri dishes. Each treatment contained equal volume of different osmotic stress solutions and distilled water as control treatment. Factorial completely randomized design (CRD) with three replications was adopted for experiment setup. Petri dishes were transferred to a controlled room $\left(25 \pm 3^{\circ} \mathrm{C}\right.$; relative humidity $60-70 \% ; 12$ hours day light) and germination data was recorded on daily basis for five days. A seed was considered to have germinated, when its radicle elongated by at least 2 $\mathrm{mm}$. For dry weight calculation, roots and shoots of 10 representative seedlings from each treatment were oven dried at $50{ }^{\circ} \mathrm{C}$ for 10 days.

\subsection{Calculation of germination parameters}

Five germination parameters were calculated as previously reported in literature. The calculation methodology for parameters 1 to 4 followed Anaya et al. (2018) and for parameter 5 Abdul-Baki and Aderson (1973).

Germination percentage $(\mathrm{GP})=\frac{\text { Number of seeds germinated in the day }}{\text { Total number of seeds }} \times 100$
Total germination percentage (TGP) $=\frac{\text { Number of seeds germinated after } 5 \text { days }}{\text { Total number of seeds }} \times 100$
Mean germination time (MGT) $=\frac{\text { Number of seeds germinated }}{\text { Day of germination }}$

Germination index $(\mathrm{GI})=$ Number of seeds germinated $\times$ Number of days after sowing Seed Vigour index $($ SVI $)=$ TGP $\times$ Dry weight

\subsection{Statistical analyses}

Two-way analysis of variance (ANOVA) was performed to compare means of respective treatments by using Statistix 10.1. Statistical analyses were conducted on mean data of three replicates with $\alpha<$ 0.05 probability level.

\section{Results and Discussion}

\subsection{Effect of drought and $S A$ on total seed germination}

To investigate possible effects of drought and SA treatments on germination, we pre-treated rice seeds with different concentrations of SA and then allowed these to germinate under different osmotic stress levels. Highly significant effects of drought, SA and their interactions were observed on total seed germination (Table 1). In absence of SA, seed germination was significantly reduced with an increase in osmotic stress level, whereas increasing SA concentration had non-significant effects on germination (Figure 1). Interestingly, varying SA concentrations imparted more pronounced effects under higher osmotic stress levels. For example, under $-0.6 \mathrm{MPa}$ osmotic stress, all SA treatments showed $90-100 \%$ germination as compared to $70 \%$ germination in control treatment (Figure 2). Similarly, $75 \mathrm{ppm}$ and $225 \mathrm{ppm}$ SA treatments also showed higher germination i.e. 93\% and $97 \%$, respectively under $-0.8 \mathrm{MPa}$ osmotic stress than respective control treatments (70\%). Whereas, less pronounced effects of different SA concentrations were observed under lower osmotic stress conditions $(-0.2$ and $-0.4 \mathrm{MPa})$. Overall, $225 \mathrm{ppm} \mathrm{SA}$ treatment showed best results under control and all osmotic stress conditions (Figure 1). Likewise, 75 ppm SA treatment demonstrated good results under medium to high stress conditions, whereas $150 \mathrm{ppm} \mathrm{SA}$ treatments showed better germination under -0.6 $\mathrm{MPa}$ osmotic stress. These results strongly suggest a potential role for $\mathrm{SA}$ in germination enhancement under water deficient conditions.

Table 1: ANOVA table summarizing two-way effect of treatments on total germination percentage of rice seeds.

\begin{tabular}{lll}
\hline Source of variation & $\begin{array}{l}\text { Degree of } \\
\text { freedom }\end{array}$ & $\begin{array}{l}\text { Mean sum of } \\
\text { square }\end{array}$ \\
\hline Drought & 4 & $834.953^{* *}$ \\
Salicylic Acid & 4 & $297.653^{* *}$ \\
Drought x Salicylic Acid & 16 & $219.445^{* *}$ \\
Error & 48 & 0.963 \\
** Highly significant at $p<0.01$. &
\end{tabular}

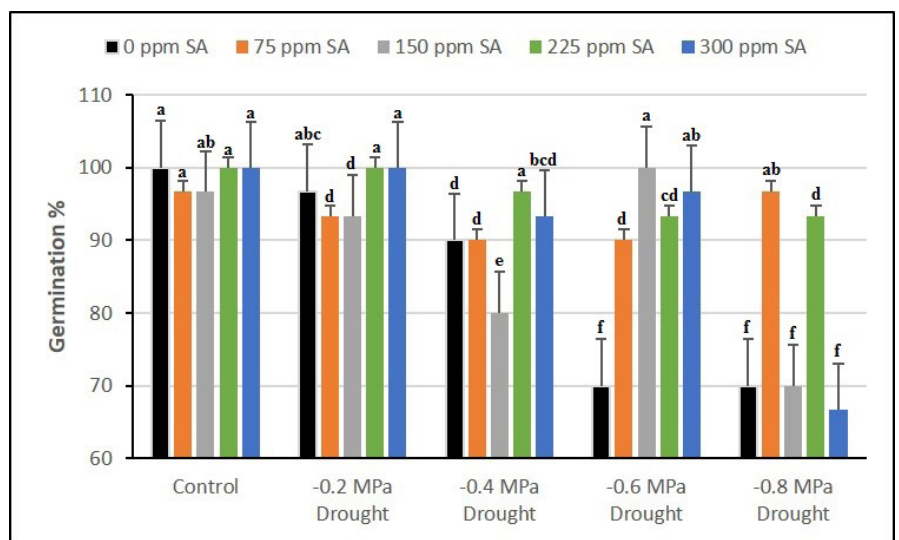

Figure 1: Total germination under drought and salicylic acid treatments.

Coloured bars indicate total germination percentage in rice seeds at $25 \pm 3{ }^{\circ} \mathrm{C}$ after five days of respective treatment application. Bars with different alphabets at their top are statistically significant $(p<0.05)$ from each other. Data are means (+ standard error) of three replicates. 


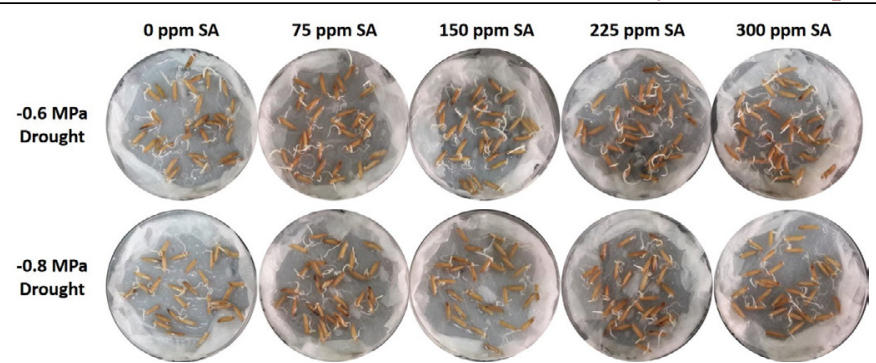

Figure 2: Effects of drought and salicylic acid treatments on total seed germination.

Rice seeds were primed with respective SA concentrations and kept overnight at $35^{\circ} \mathrm{C}$. Then hydrated seeds were allowed to germinate at $25 \pm 3{ }^{\circ} \mathrm{C}$ in darkness under different osmotic stress levels. The picture was taken at the end of experiment after five days of sowing.

\subsection{Effect of $S A$ on germination process under water deficient conditions}

To determine at which time point significant differences occur between germination of SA treated and non-treated seeds, we collected germination data from all osmotic stress and control treatments on daily basis and generated germination speed curve using line graphs (Figure 3). Under normal condition, germination started after $1^{\text {st }}$ day $(3 \%)$ and reached maximum after $4^{\text {th }}$ day $(100 \%)$ in control treatment (Figure 3A). In SA treatments, germination also started after $1^{\text {st }}$ day (0-3\%) but reached maximum (97$100 \%)$ after $5^{\text {th }}$ day. Interestingly, germination rate was lower in all SA treatments after $2^{\text {nd }}, 3^{\text {rd }}$ and $4^{\text {th }}$ day as compared with control treatment.Under osmotic stress conditions, germination was delayed and significantly reduced $(\mathrm{p}<0.01)$ with an increase in the stress level (Figure 3B). Significant reductions were observed after 2-4 days among germination rates of different osmotic stress treatments. These results indicate that $2^{\text {nd }}, 3^{\text {rd }}$ and $4^{\text {th }}$ days are critical for germination process, as during this period any differences due to favourable or harmful conditions become more apparent between experimental treatments.
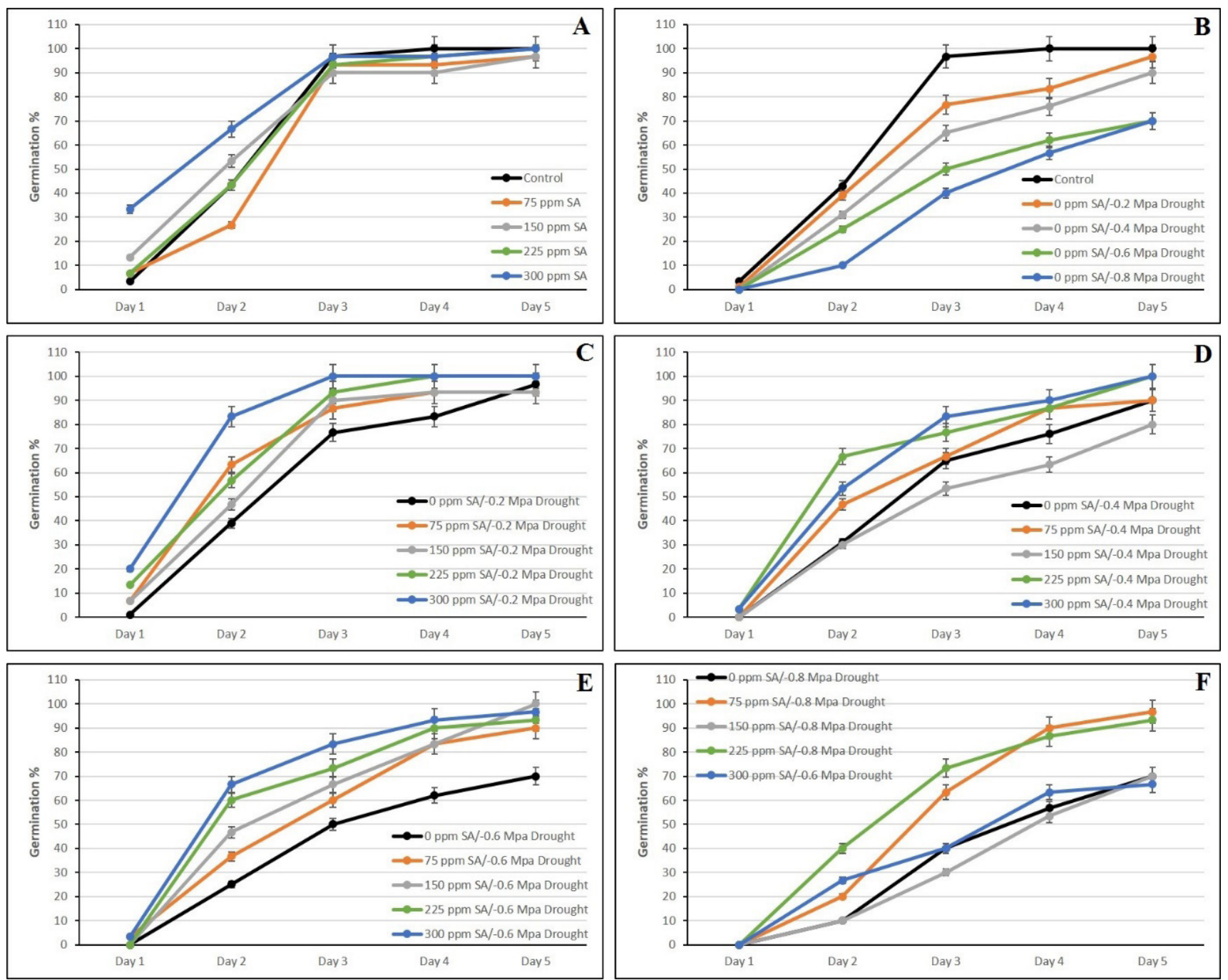

Figure 3: Daily germination under drought and salicylic acid treatments.

Daily germination percentage of rice seeds in different concentrations of SA (A), drought (B) and their interactive treatments $(\mathrm{C}-\mathrm{F})$ at $25 \pm 3{ }^{\circ} \mathrm{C}$ in darkness. Data are means $(\% \pm$ S.E) of three replicates.

Journal of Innovative Sciences

June 2021 | Volume 7 | Issue 1 | Page 155 

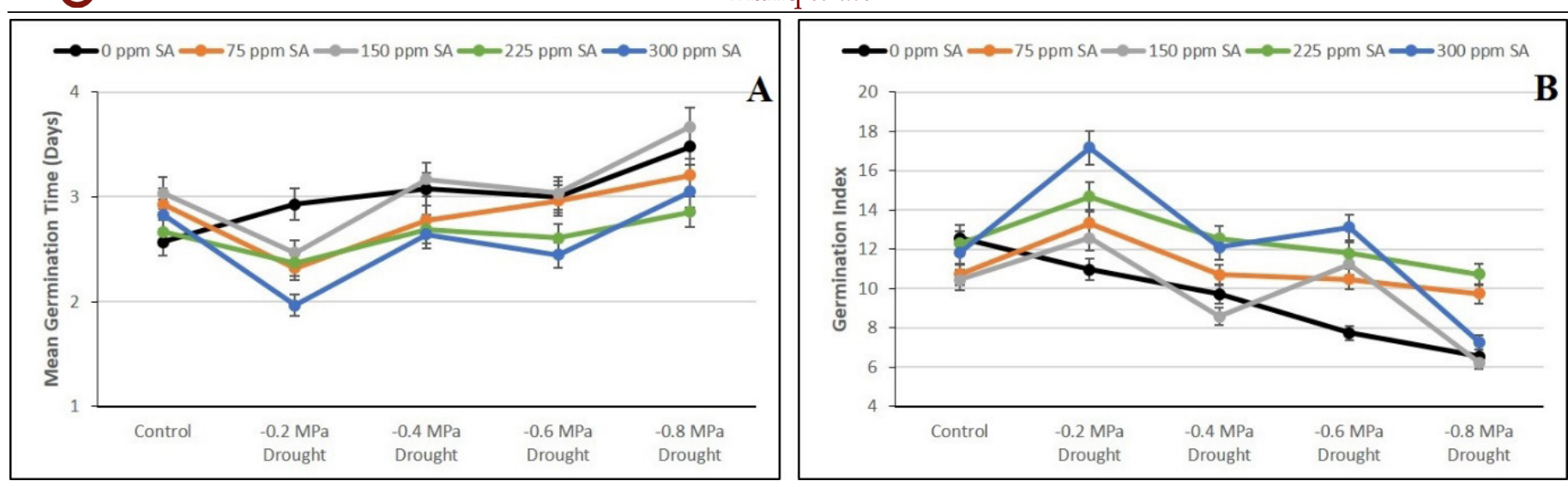

Figure 4: Effect of SA concentrations on mean germination time and germination index at different osmotic stress levels.

Mean germination time (A) and germination index (B) of rice seeds after five days of drought, SA and their interactive treatments. Data are means $(\% \pm$ S.E $)$ of three replicates.

The combination of SA treatments with osmotic stress levels imparted beneficial effects on germination process. Under low osmotic stress level $(-0.2 \mathrm{MPa})$, all SA treatments showed high germination rates after 1-4 days than respective control treatment (Figure 3C). Under medium osmotic stress levels (-0.4-0.6 MPa), at least two SA treatments (225 $\mathrm{ppm}$ and $300 \mathrm{ppm}$ ) showed enhanced germination during 2-4 days as compared to respective control treatments (Figure 3D and 3E). Similarly, under high osmotic stress level (-0.8 MPa), $75 \mathrm{ppm}$ and $225 \mathrm{ppm}$ SA treatments resulted in improved germination during 2-5 days as compared with control treatment (Figure 3F). Remarkably, all SA treatments exhibited highly significant beneficial effects on germination process after $2-5$ days under $-0.6 \mathrm{MPa}$ osmotic stress. Likewise, $75 \mathrm{ppm}$ and $225 \mathrm{ppm} \mathrm{SA}$ treatments also demonstrated highly significant effects under -0.8 $\mathrm{MPa}$ osmotic stress. These results strongly indicate that beneficial effects of SA treatments alleviated inhibitory effects of different osmotic stress levels after 2-4 days of stress applications.

\subsection{Effect of $S A$ on mean germination time and germination index}

Increase in osmotic stress levels delayed the germination process, as MGT and GI were increased and decreased, respectively. Under normal conditions, control treatment had lowest MGT (2.57 days) and reached maximum (3.48 days) under $-0.8 \mathrm{MPa}$ osmotic stress (Figure 4A). All SA treatments exhibited higher MGT in absence of osmotic stress as compared with control treatment. Whereas, under stress conditions, three SA treatments (75 ppm, 225 ppm and $300 \mathrm{ppm}$ ) showed less MGT as compared to respective control treatments. Interestingly, MGT for $225 \mathrm{ppm}$ and $300 \mathrm{ppm} \mathrm{SA}$ treatments were significantly lower than control treatments under all stress levels. Furthermore, MGT ranged between 2-4 days during control and stress treatments. In contrast to MGT, GI demonstrated decreasing trend with an increase in osmotic stress levels (Figure 4B). Under normal conditions, control treatment had high GI than all SA treatments. Under $-0.2 \mathrm{MPa}$ and -0.6 $\mathrm{MPa}$ osmotic stresses, all SA treatments exhibited higher GI than respective controls. Likewise, under all stress conditions, $75 \mathrm{ppm}, 225 \mathrm{ppm}$ and $300 \mathrm{ppm}$ SA treatments showed higher GI as compared to respective controls. Collectively, these results strongly support our data and indicate advantageous influence of SA treatments on germination process under stressful conditions.

\subsection{Effect of drought and $S A$ treatments on seed vigour index}

To examine physiological effects of drought and SA treatments on SVI, we also recorded dry matter weight data from 10 representative seedlings as mentioned in material and method section. The SVI first significantly increased and then decreased as compared to control with an increase in osmotic stress levels (Figure 5). Under normal conditions, 300 ppm SA treatment exhibited higher SVI than control treatment. Likewise, $225 \mathrm{ppm} \mathrm{SA}$ treatment also showed comparable SVI with control treatment. Interestingly, under all osmotic stress levels, $75 \mathrm{ppm}$, $225 \mathrm{ppm}$ and $300 \mathrm{ppm}$ SA treatments demonstrated significantly high or comparable SVI when compared with respective controls. Whereas, at medium to high osmotic stress levels (-0.6 MPa and $-0.8 \mathrm{MPa})$, 
all SA treatments showed significantly higher SVI than controls (Figure 5). All these results collectively indicate a strong beneficial effect of SA treatments on seed germination process and crop establishment under water deficient environments.

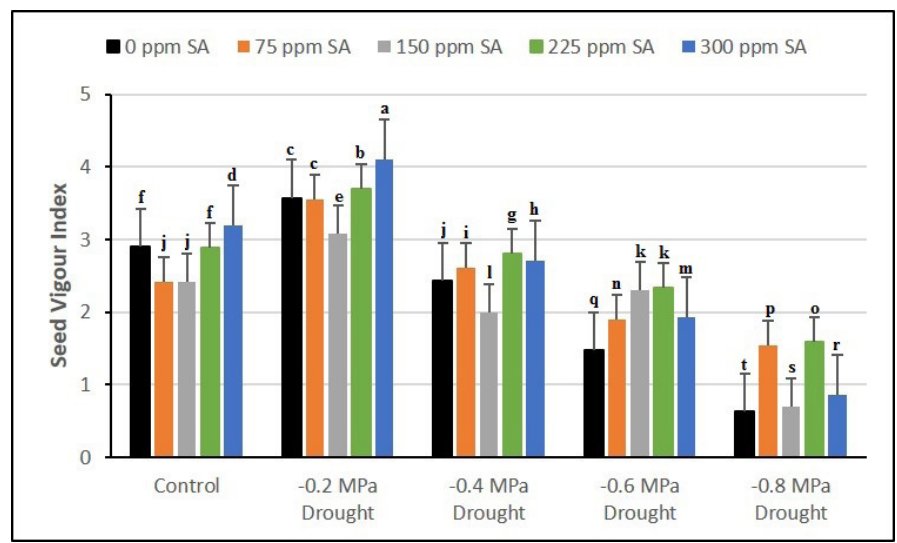

Figure 5: Effect of drought and salicylic acid treatments on seed vigour index.

Coloured bars indicate seed vigour index value of rice seeds at respective treatments. Bars with different alphabets on their top are statistically significant $(p<0.05)$ from each other. Data are means $(+$ S.E) of three replicates.

New interventions in DSR system are required for enhancement of seed germination and early plant growth to ensure rice production on sustainable basis. The beneficial effects of SA in biotic and abiotic stress tolerance in crop plants have been well documented. In this study, we investigated possible effects of SA on rice seed germination under induced drought stress conditions.

Germination process starts after changes in seed water contents and completes with radicle elongation (Ali and Elozeiri, 2017). During germination, drought stress causes reduction in water availability and subsequently reduces germination indices (Islam et al., 2018). In this study, germination was also reduced significantly with an increase in water stress level (Figures 1 and 2). In presence of SA, germination was significantly improved under medium to high drought conditions. However, no significant differences in germination indices occurred between control and SA treatments in absence of water stress. These results are in accordance with Anaya et al. (2018) and indicate that inhibitory effects of drought stress on germination indices are reversed by SA treatments. Thus, a clear evidence between SA based seed priming and germination enhancement under drought stress have been established in rice which could address poor crop establishment issues in DSR system.
Increasing evidences have suggested that drought stress delays the germination process (Pandey and Shukla, 2015) and 2-4 days after sowing are most critical of radicle elongation (Rice Knowledge Bank, 2021; Ella et al., 2011) because any differences in germination indices due to experimental conditions are more clear during this period. This hypothesis prompted us to investigate drought and SA effects on daily germination rates. Here, germination was delayed in presence of water stress and absence of SA treatments, whereas maximum germination was achieved after 4 days in control treatment (Figure $3 \mathrm{~A}$ and $3 \mathrm{~B}$ ). In drought, SA and their interactive treatments, germination differences were more prominent after $2-4$ days (Figure $3 \mathrm{~A}-\mathrm{F}$ and $4 \mathrm{~A}$ ). These results strongly suggest that $2-4$ days after sowing are critical and a quick germination report is available after this period which could help in subsequent decision making, if poor germination is observed.

Recent studies have reported drought and SA mediated regulation of growth and development in crop plants. Chen et al. (2020) showed that drought stress intensity significantly effects rice seedlings growth as compared with drought duration. Similarly, Sohag et al. (2020) demonstrated that exogenous hydrogen peroxide $\left(\mathrm{H}_{2} \mathrm{O}_{2}\right)$ and $\mathrm{SA}$ applications significantly improve rice seedlings growth under drought conditions. In this research, varying SA concentrations also had significant effects on SVI (Figure 5). Under medium to high osmotic stress levels, SVI of SA treated seeds was at par than non-treated seeds. Interestingly, SVI of all treatments under $-0.2 \mathrm{MPa}$ osmotic stress was surprisingly improved as compared with control and other osmotic stress levels. The unexpected increase in SVI might indicate a 'drought-escape strategy' in rice seeds under low osmotic stress level. This kind of escape strategy is also reported during different abiotic stresses such as chilling, drought, salinity and high temperature in crop plants (Franks, 2011; Kooyers, 2015; Gull et al., 2019). Collectively, these results demonstrate a positive effect of SA on seed germination and seedlings health and provide new insights into drought response mechanism at early growth stage.

Different SA concentrations impart dissimilar effects on germination indices under varying stress levels and a lack of harmony exists in literature regarding ideal dose of SA for optimal plant growth under normal 
and stress conditions (Tavares et al., 2014; Shatpathy et al., 2018; Ahmed et al., 2020). In absence of stress, SA reveals non-significant effects on germination (Figure 2). On the contrary, it gives optimal results under stressful conditions. However, change in SA concentration is often linked with arbitrary change in germination indices, thus making it very difficult to identify an optimal dose which equally performs under changing environments. In this study, we identified osmotic stress level specific SA doses for germination improvement. Seed priming with 225 ppm SA exhibited significantly high germination indices under all osmotic stress levels and it also showed non-significant difference when compared with control treatment (Figures 2, 3, 4 and 5). Similarly, 75 ppm SA treatment demonstrated high germination under medium to high osmotic stress levels and $150 \mathrm{ppm}$ SA treatments showed best results under medium osmotic stress level. Collectively, these results indicate that seed priming with 225 ppm SA concentration is ideal under all environments and should be recommended in DSR system to improve crop establishment and sustainable production.

\section{Conclusion and Recommendations}

Cumulative results of this study demonstrated inhibitory effects of induced drought stress on rice seed germination. However, pre-treatment of seed with different SA concentrations alleviated inhibitory effects of drought stress. Varying SA concentrations had beneficial effects on seed germination process and seedlings vigour index. Overall, $225 \mathrm{ppm}$ SA concentration demonstrated better germination indices under all stress and respective control treatments. Based on the results presented in this study, pre-treatment of rice seed with $225 \mathrm{ppm}$ SA concentration is recommended for enhanced germination and early crop establishment in DSR system.

\section{Novelty Statement}

To address poor germination issues in DSR system, we investigated the beneficial effects of seed priming with salicylic acid under induced drought stress conditions. Results demonstrated that $225 \mathrm{ppm}$ concentration of SA was ideal and should be recommended for enhanced germination under DSR system.

\section{Author's Contribution}

CMR, QR, AR and MS conceived and designed the experiment. $\mathrm{QR}, \mathrm{MH}$ and WS performed the experiment. AR, $\mathrm{MH}$ and $\mathrm{WS}$ data collection. $\mathrm{CMR}$, $\mathrm{QR}, \mathrm{AR}, \mathrm{SSA}$ and MS data analysis. QR and AR writing original draft. CMR, SI, THA, SSA and MS review and editing. The final manuscript was ultimately perused, scrutinized and approved for final submission by all the authors.

\section{Conflict of interest}

The authors have declared no conflict of interest.

\section{References}

Abdul-Baki, A.A., and Anderson, J.D., 1973. Vigor determination in soybean seed by multiple criteria 1. Crop Science, 13(6):630-633.https://doi. org/10.2135/cropsci1973.0011183X001300060013x

Ahmed, W., Imran, M., Yaseen, M., Haq, T., Jamshaid, M.U., Rukh, S., and Khan, M.A., 2020. Role of salicylic acid in regulating ethylene and physiological characteristics for alleviating salinity stress on germination, growth and yield of sweet pepper. PeerJ., 8: e8475. https:/doi. org $/ 10.7717 /$ peerj. 8475

Ali, A.S., and Elozeiri, A.A., 2017. Metabolic processes during seed germination. Advances in Seed Biology, pp. 141-166. https://doi.org/10.5772/ intechopen.70653

Anaya, F., Fghire, R.,Wahbi,S., and Loutfi,K.,2018. Influence of salicylic acid on seed germination of Vicia faba L. under salt stress. Journal of the Saudi Society of Agricultural Sciences, 17(1): 1-8. https://doi.org/10.1016/j.jssas.2015.10.002

Budak, H., Hussain, B., Khan, Z., Ozturk, N.Z., and Ullah, N., 2015. From genetics to functional genomics: Improvement in drought signaling and tolerance in wheat. Frontiers in Plant Science, 6: 1012. https://doi.org/10.3389/ fpls.2015.01012

Chen, Z.K., Xu, W.W., Nie, J., Khan, A., Cao, C.G., and Li, P., 2020. Drought stress intensity, duration and its resistance impact on rice (Oryza sativa L.) seedling. Applied Ecology and Environmental Research, 18(1): 469-486. https:// doi.org/10.15666/aeer/1801_469486

El-Mokhtar, S.M., Samb, A., Moufid, A.O., Boukhary, A.O.M.S., and Djeh, T.K.O., 2015. Effectofdifferentlevels of salinityongermination 
and early seedling growth of three rice varieties cultivated in Mauritania. International Journal of Agriculture and Crop Sciences, 8(3): 346-349.

Ella, E.S., Dionisio-Sese, M.L., and Ismail, A.M., 2011. Seed pre-treatment in rice reduces damage, enhances carbohydrate mobilization and improves emergence and seedling establishment under flooded conditions. $A \circ B$ Plants, 2011. https://doi.org/10.1093/aobpla/plr007

Franks, S.J., 2011. Plasticity and evolution in drought avoidance and escape in the annual plant Brassica rapa. New Phytologist, 190(1): 249-257. https://doi.org/10.1111/j.1469-8137.2010.03603.x

Fróna, D., Szenderák, J., and Harangi-Rákos, M., 2019. The challenge of feeding the world. Sustainability, 11(20): 5816. https://doi. org/10.3390/su11205816

Fukagawa, N.K., and Ziska, L.H., 2019. Rice: Importance for global nutrition. Journal of nutritional science and vitaminology, 65(Supplement): S2-S3. https:// doi.org/10.3177/jnsv.65.S2

Gull, A., Lone, A.A., and Wani, N.U.I., 2019. Biotic and abiotic stresses in plants. Abiotic and Biotic Stress in Plants, pp. 1-19. https://doi.org/10.5772/ intechopen.85832

Guo, B., Liang, Y.C., Zhu, Y. G., and Zhao, F.J., 2007. Role of salicylic acid in alleviating oxidative damage in rice roots (Oryza sativa) subjected to cadmium stress. Environmental Pollution, 147(3): 743-749. https://doi. org/10.1016/j.envpol.2006.09.007

Hussain, M., Farooq, M., and Lee, D.J., 2017. Evaluating the role of seed priming in improving drought tolerance of pigmented and non冈pigmented rice. Journal of Agronomy and Crop Science, 203(4): 269-276. https://doi. org/10.1111/jac.12195

Islam, M.M., Kayesh, E., Zaman, E., Urmi, T.A., and Haque, M.M., 2018. Evaluation of rice (Oryza sativa L.) genotypes for drought tolerance at germination and early seedling stage. The Agriculturists, 16(1): 44-54. https:// doi.org/10.3329/agric.v16i1.37533

Iqbal, S., Khalid, U.B., Awan, T.H., Iqbal, N., Saleem, M.U., Iram, A., and Ahmad, N., 2019. Qualitative response of super basmati rice to different nitrogen levels under varying rice ecosystem. African Journal of Agricultural Research, 14(9): 548-558.

Iqbal, S., Iqbal, N., Khalid, U.B., Saleem, M.U.,
Iram, A., Rizwan, M., and Awan, T.H., 2021. Growth, yield and economic analysis of dry-seeded basmati rice. Sarhad Journal of Agriculture, 37(1): 200-208. https://doi. org/10.17582/journal.sja/2021/37.1.200.208

Jan, M., Shinwari, K.I., Shah, G., Khan, M.H.U., Ullah, S., Hameed, A., and Malook, I., 2015. Consequences of short-term low temperature stress on physiological and biochemical aspects of rice (Oryza sativa L.). Scientia Agriculturae, 10(1):1-14.https://doi.org/10.15192/ PSCP.SA.2015.10.1.114

Khan, M.S., Akther, T., Ali, D.M., and Hemalatha, S., 2019. An investigation on the role of salicylic acid alleviate the saline stress in rice crop (Oryza sativa L). Biocatalysis and Agricultural Biotechnology, 18: 101027. https:// doi.org/10.1016/j.bcab.2019.101027

Kooyers, N.J., 2015. The evolution of drought escape and avoidance in natural herbaceous populations. Plant Science, 234: 155-162. https:// doi.org/10.1016/j.plantsci.2015.02.012

Kurniasih, B., Greenway, H., and Colmer, T.D., 2013. Tolerance of submerged germinating rice to $50-200 \mathrm{mM} \mathrm{NaCl}$ in aerated solution. Physiologia Plantarum, 149(2): 222233. https://doi.org/10.1111/ppl.12029

Liu, H., Hussain, S., Zheng, M., Peng, S., Huang, J., Cui, K., and Nie, L., 2015a. Dry direct-seeded rice as an alternative to transplanted-flooded rice in Central China. Agronomy for Sustainable Development, 35(1): 285-294. https://doi. org/10.1007/s13593-014-0239-0

Liu, S.J., Xu, H.H., Wang, W.Q., Li, N., Wang, W.P., Møller, I.M., and Song, S.Q., 2015b. A proteomic analysis of rice seed germination as affected by high temperature and ABA treatment. Physiologia Plantarum, 154(1): 142161. https://doi.org/10.1111/ppl.12292

Matsushima, K.I., and Sakagami, J.I., 2013. Effects of seed hydropriming on germination and seedling vigor during emergence of rice under different soil moisture conditions. American Journal of Plant Sciences, 2013. https://doi. org/10.4236/ajps.2013.48191

Mohamed, H.I., El-Shazly, H.H., and Badr, A., 2020. Role of salicylic acid in biotic and abiotic stress tolerance in plants. Plant Phenolics in Sustainable Agriculture Springer, Singapore. pp. 533-554. https://doi.org/10.1007/978-981-154890-1_23 
Pandey, V., and Shukla, A., 2015. Acclimation and tolerance strategies of rice under drought stress. Rice Science, 22(4): 147-161. https://doi. org/10.1016/j.rsci.2015.04.001

Rao, A.N., Wani, S.P., Ramesha, M.S., and Ladha, J.K., 2017. Rice production systems. Rice Production Worldwide Springer, Cham. pp. 185205. https://doi.org/10.1007/978-3-319-47516-5_8

Rice Knowledge Bank, 2021. Available at http:// www.knowledgebank.irri.org/training/factsheets/management-of-other-crop-problemsfact-sheet-category/measuring-seedgermination-fact-sheet (accessed 15 February 2021).

Rivas-San Vicente, M., and Plasencia, J., 2011. Salicylic acid beyond defence: its role in plant growth and development. Journal of Experimental Botany, 62(10): 3321-3338. https://doi.org/10.1093/jxb/err031

Sarfraz, M., Hussain, S., Ijaz, M., Nawaz, A., Yasir, T.A., Sher, A., and Ahmad, S., 2019. Abiotic stress tolerance in plants by priming and pretreatments with phytohormones. Priming and Pretreatment of Seeds and Seedlings Springer, Singapore. pp. 447-457. https://doi. org/10.1007/978-981-13-8625-1_22

Sharma, B., and Parikh, M., 2020. Seed priming: An emerging technology to impart abiotic stress tolerance in rice. International Journal of Communication Systems, 8(2): 619-623. https:// doi.org/10.22271/chemi.2020.v8.i2j.8837

Shatpathy, P., Kar, M., Dwibedi, S.K., and Dash, A., 2018. Seed priming with salicylic acid improves germination and seedling growth of rice (Oryza sativa L.) under PEG-6000 induced water stress. Int.J. Curr. Microbiol. Appl. Sci., 7(10): 907-924. https://doi.org/10.20546/ ijcmas.2018.710.101
Sheteiwy, M., Shen, H., Xu, J., Guan, Y., Song, W., and $\mathrm{Hu}, \mathrm{J} ., 2017$. Seed polyamines metabolism induced by seed priming with spermidine and 5-aminolevulinic acid for chilling tolerance improvement in rice (Oryza sativa L.) seedlings. Environmental and Experimental Botany, 137: 58-72. https://doi.org/10.1016/j. envexpbot.2017.02.007

Sohag, A.A.M., Tahjib-U1-Arif, M., Brestic, M., Afrin, S., Sakil, M.A., Hossain, M.T., and Hossain, M.A., 2020. Exogenous salicylic acid and hydrogen peroxide attenuate drought stress in rice. Plant Soil and Environment, 66(1): 7-13. https://doi.org/10.17221/472/2019-PSE

Tavares, L.C., Rufino, C.A., Oliveira, S.D., Brunes, A.P., and Villela, F.A., 2014. Treatment of rice seeds with salicylic acid: seed physiological quality and yield. Journal of Seed Science, 36(3): 352-356. https://doi.org/10.1590/2317$1545 \mathrm{v} 36 \mathrm{n} 3636$

Wang, W., Peng, S., Chen, Q. Mei, J., Dong, H., and Nie, L., 2016. Effects of pre-sowing seed treatments on establishment of dry directseeded early rice under chilling stress. $A O B$ Plants, pp. 8.https://doi.org/10.1093/aobpla/plw074 Wei, L.X., Lv, B.S., Wang, M.M., Ma, H.Y., Yang, H.Y.,Liu, X.L., and Liang, Z.W., 2015.Priming effect of abscisic acid on alkaline stress tolerance in rice (Oryza sativa L.) seedlings. Plant Physiology and Biochemistry, 90: 50-57. https:// doi.org/10.1016/j.plaphy.2015.03.002

Zheng, M., Tao, Y., Hussain, S., Jiang, Q., Peng, S., Huang, J., and Nie, L., 2016. Seed priming in dry direct-seeded rice: consequences for emergence, seedling growth and associated metabolic events under drought stress. Plant Growth Regulation, 78(2): 167-178. https://doi. org/10.1007/s10725-015-0083-5 